\title{
Preoperative assessment of para- aortic lymph node metastasis in patients with pancreatic cancer
}

\section{$\operatorname{AUTHOR}(S)$ :}

Imai, Hisashi; Doi, Ryuichiro; Kanazawa, Hiroyuki; Kamo, Naoko; Koizumi, Masayuki; Masui, Toshihiko; Iwanaga, Yasuhiro; ... Takada, Yasutsugu; Isoda, Hiroyoshi; Uemoto, Shinji

\section{CITATION:}

Imai, Hisashi ... [et al]. Preoperative assessment of para-aortic lymph node metastasis in patients with pancreatic cancer. International journal of clinical oncology / Japan Society of Clinical Oncology 2010, 15(3): 294-300

\section{ISSUE DATE:}

2010-06

URL:

http://hdl.handle.net/2433/126729

\section{RIGHT:}

The original publication is available at www.springerlink.com; この論文 は出版社版でありません。引用の際には出版社版をご確認ご利用くだ さい。; This is not the published version. Please cite only the published version. 


\section{Preoperative assessment of para-aortic lymph node metastasis in patients with pancreatic cancer}

Hisashi Imai ${ }^{1}$, Ryuichiro Doi ${ }^{1}$, Hiroyuki Kanazawa ${ }^{1}$, Naoko Kamo ${ }^{1}$, Masayuki Koizumi ${ }^{1}$, Toshihiko Masui ${ }^{1}$, Yasuhiro Iwanaga ${ }^{1}$, Yoshiya Kawaguchi ${ }^{1}$, Yasutsugu Takada ${ }^{1}$, Hiroyoshi Isoda ${ }^{2}$, and Shinji Uemoto ${ }^{1}$

1) Department of Hepato-Biliary-Pancreatic Surgery and Transplantation, Kyoto University, Kyoto, Japan

2) Department of Diagnostic Imaging and Nuclear Medicine, Kyoto University, Kyoto, Japan

\section{Correspondence:}

Ryuichiro Doi, MD

Department of Hepato-Biliary-Pancreatic Surgery and Transplantation, Kyoto University

54 Shogoinkawaracho, Sakyoku

Kyoto 606-8507, Japan

TEL: +81-75-751-4323, FAX: +81-75-751-4877

E-mail: doir@kuhp.kyoto-u.ac.jp

Running Title: Para-aortic lymph node metastasis in patients with pancreatic cancer Key words: invasive ductal carcinoma of the pancreas; para-aortic lymph node metastasis; diagnostic imaging 


\begin{abstract}
$\underline{\text { Backgrounds }}$

Para-aortic lymph node (PALN) metastasis is an important prognostic factor in patients with pancreatic cancer, but accurate preoperative diagnosis is difficult. The aim of this study was to assess the accuracy of diagnosing PALN by computed tomography (CT), magnetic resonance imaging (MRI), and ${ }^{18}$ F-fluorodeoxyglucose positron emission tomography (FDG-PET).
\end{abstract}

\title{
$\underline{\text { Methods }}$
}

From August 2005 to July 2008, 119 patients with invasive ductal adenocarcinoma of the pancreas were included in this study. PALNs with a longer diameter $>10 \mathrm{~mm}$ on CT or MRI were suspected to be involved by metastasis, while ${ }^{18}$ F-fluorodeoxyglucose (FDG) uptake exceeding that of the adjacent normal tissue was considered to be positive for metastasis on FDG-PET studies. The imaging findings were compared with the pathological diagnosis of PALN metastasis.

\section{$\underline{\text { Results }}$}

PALN dissection was performed in 71 patients (60.0\%). Although histopathological examination revealed metastasis in 6 patients (8.5\%), none of these patients were positive in any of the preoperative imaging studies. The longer diameter, the shorter diameter, the ratio of the two diameters, and the calculated lymph node volume showed no significant differences between patients with and without PALN metastasis.

\section{$\underline{\text { Conclusions }}$}

Preoperative detection of PALN metastasis in patients with pancreatic cancer is very difficult. Intraoperative histopathological examination of frozen sections is necessary if radical resection is 
$-3-$

contemplated.

\section{Mini-Abstract}

Para-aortic lymph node metastasis is an important prognostic factor in patients with pancreatic cancer, but accurate preoperative diagnosis is difficult. Intraoperative histopathological examination is necessary if radical resection is contemplated. 


\section{Introduction}

Even in the 21st century, pancreatic cancer still has a dismal prognosis. The overall median survival time is 8.6 months and 5-year survival rate is $9.7 \%$ for Japanese patients with invasive ductal adenocarcinoma ${ }^{1}$. Complete surgical resection is the only potentially curative treatment available, but less than $20 \%$ of all patients have resectable disease and the 5 -year overall survival of resectable patients is still only $10-20 \%^{2}$. Although extended radical surgery has been performed for various stages of pancreatic cancer, extended lymphadenectomy has not been shown to prolong survival compared with standard lymphadenectomy ${ }^{1,3}$. The presence of PALN metastasis is reported to be an important adverse prognostic factor in patients with pancreatic cancer ${ }^{4}$. Therefore, preoperative evaluation of the PALN status is one of the most important factors when deciding whether pancreatic resection should be performed or not.

It was reported that only 33\% of PALN metastases are suspected preoperatively or perioperatively ${ }^{5}$. The ability of preoperative imaging modalities to evaluate local resectability and to identify metastatic disease has increased substantially, and helical computed tomography (CT), magnetic resonance imaging (MRI), endoscopic ultrasonography (EUS), endoscopic retrograde cholangiopancreaticography (ERCP), and ${ }^{18}$ F-fluorodeoxyglucose positron emission tomography (FDG-PET) are commonly used at present. Among these modalities, CT and EUS are considered to be the most useful with respect to staging of pancreatic cancer ${ }^{6,7}$. However, he detection of small liver metastases and peritoneal metastases remains limited ${ }^{8}$. Similarly, the sensitivity of these imaging methods for lymph node metastasis is low (EUS, 67\%; CT, 55\%; MRI, 75\%), and lymph node metastasis of pancreatic cancer remains difficult to detect preoperatively ${ }^{7}$.

At our hospital, PALN dissection precedes other procedures in patients with pancreatic cancer because we do not perform radical pancreatectomy if PALN metastasis is detected by intraoperative 
frozen section diagnosis. Therefore, if we could determine the presence of PALN metastasis before laparotomy, this would be beneficial for our patients.

Accordingly, the purpose of the present study was to examine the diagnostic accuracy of preoperative imaging modalities (CT, MRI, and FDG-PET) for PALN metastasis in patients with invasive carcinoma of the pancreas. Based on our findings, it remains very difficult to detect PALN metastasis, even by the most recent imaging methods.

\section{Methods}

\section{$\underline{\text { Patients }}$}

Patients from our hospital in whom a histopathological diagnosis of invasive ductal adenocarcinoma of the pancreas was made between August 2005 and July 2008 were included in this study. Patients with other pancreatic malignancies, such as intraductal papillary mucinous neoplasms or neuroendocrine tumors, were excluded.

Patients routinely underwent preoperative contrast-enhanced helical CT, MRI, and FDG-PET to assess the resectablity and stage of the tumor according to the Classification of Pancreatic Carcinoma by the Japan Pancreas Society (JPS) ${ }^{9}$ and the TNM classification of the Union International Contra la Cancrum (UICC) ${ }^{10}$.

\section{$\underline{\text { Surgical procedure }}$}

If the tumor was considered to be resectable as a result of preoperative assessment, the patient underwent laparotomy. Resectable disease was defined as the absence of distant metastasis and no infiltration of major arteries, such as the common hepatic, celiac, and superior mesenteric arteries. Invasion of the portal vein (PV), superior mesenteric vein (SMV), or splenic vein was not a 


\section{$-6-$}

contraindication to resection, and such vessels were resected if necessary.

At the start of surgery, $50 \mathrm{ml}$ of warm saline was infused into the Pouch of Douglas and was collected for intraoperative cytological examination. Liver metastasis and peritoneal dissemination were assessed by inspection and palpation. If metastasis or dissemination was suspected, the lesions were investigated by intraoperative frozen section examination. Next, dissection of the PALNs between the celiac axis and the inferior mesenteric artery was performed. This involved total resection of the lymph nodes belonging to stations $16 \mathrm{a} 2$ and $16 \mathrm{~b} 1{ }^{11}$. These lymph nodes were also submitted for intraoperative frozen section examination.

If tumor cells were found by cytological examination, or if liver metastasis, peritoneal dissemination, or PALN metastasis was detected, radical resection was abandoned and we selected palliative surgery plus postoperative systemic chemotherapy. Otherwise, pancreaticoduodenectomy (with or without pylorus preservation), distal pancreatectomy, or total pancreatectomy was performed according to the tumor location.

Postoperatively, the formalin-fixed resected specimens with attached lymph nodes were examined histopathologically for definitive tumor staging. Intraoperative samples were also examined again after formalin fixation.

\section{Preoperative CT, MRI and FDG-PET}

CT scanning was performed with a 64-detector row scanner (Aquilion Toshiba Medical, Tokyo, Japan) at a tube voltage of $120 \mathrm{kV}, 200-250$ mAs per 360 degrees, gantry rotation time of 0.5 seconds, detector collimation of $64 \times 0.5 \mathrm{~mm}$, and table feed of $26.5 \mathrm{~mm}$ per rotation. Contrast medium (Iopamiron: Bayer-Schering Pharma, Germany; Iomeron: Bracco-Eisai, Tokyo, Japan) was injected intravenously over 30 seconds at a dose of $2 \mathrm{ml} / \mathrm{kg}$ using a power injector (Auto Enhance A-50; Nemoto Kyorindou, Tokyo, Japan). In all cases, RealPrep was used to determine the timing 


\section{$-7-$}

of scans and the threshold was set at $200 \mathrm{HU}$ attenuation in the lower descending aorta. The scanning delay for the late arterial and equilibrium phases was 25 and 60 seconds, respectively.

MRI was performed with a 1.5 T super conducting MR unit (Magnetom Symphony, Siemens, Germany). Three types of images (breath-holding T1-weighted gradient-echo images in the axial plane [GE, slice thickness, $6 \mathrm{~mm}$; slice gap, $1 \mathrm{~mm}$ ], breath-holding true FISP images in the coronal plane [slice thickness, $5 \mathrm{~mm}$; slice gap, $0 \mathrm{~mm}$ ], and respiration-triggered T2-weighted turbo spin-echo images in the axial plane [slice thickness, $6 \mathrm{~mm}$; slice gap, $1 \mathrm{~mm}$ ]) were obtained.

FDG-PET was performed by using a high-resolution, whole-body PET scanner with an 18-ring detector (Advance; General Electric Medical Systems, Milwaukee, WI). All patients received an intravenous injection of ${ }^{18}$ F-FDG $(296 \pm 74 \mathrm{MBq})$ at 50 minutes before the acquisition of whole-body PET images was started. Emission images were acquired for 3 minutes per bed position, and each post-emission transmission scan was acquired for 1 minute per position. Whole-body scanning (from the face to upper thighs) was performed in each patient by using five or six bed positions according to their height. Data were reconstructed by the ordered subsets expectation maximization method (OSEM) using 16 subsets, 3 iterations, and a 128 x 128 array.

\section{Image analysis}

All images were interpreted by at least two experienced radiologists who used all available clinical information and had correlative conventional imaging for anatomic guidance (Figure 1).

On CT and MRI, the shorter and longer diameters of the largest PALN were measured in both axial and coronal images. PALNs with a longer diameter $>10 \mathrm{~mm}$ were suspected to be involved by metastasis. The estimated PALN volume was calculated as 0.5 x L x S2 [L: longer diameter, S: shorter diameter].

On PET images, regions of interest (ROIs) were manually defined on transaxial tomograms for 
$-8-$

semiquantitative analysis of ${ }^{18} \mathrm{~F}$-FDG uptake and experienced nuclear medicine physicians read the PET images. When a PALN showed increased ${ }^{18}$ F-FDG accumulation compared with the surrounding tissues that was not related to normal physiologic uptake, it was considered to be positive for malignancy. Sites of increased ${ }^{18}$ F-FDG uptake were not considered to indicate metastasis if related to a known nonmalignant process or to the physiologic biodistribution of ${ }^{18}$ F-FDG.

$\underline{\text { Statistical analysis }}$

Results are expressed as the mean \pm SD. Differences between two groups were analyzed by using the $\chi 2$ test. Statistical analysis was performed with JMP software (SAS institute, Cary, NC) and $\mathrm{P}<0.05$ was considered to indicate statistical significance. 


\section{Results}

\section{$\underline{\text { Patient Profile }}$}

This study included 119 patients with a histological diagnosis of invasive ductal carcinoma of the pancreas from August 2005 to July 2008. Their clinical, surgical, and histopathological findings are presented in Tables 1 and 2.

Curative resection was performed in 85 patients (71.4\%) and palliative surgery, including duodenal and/or biliary bypass, was done in 17 patients (14.3\%). Surgical intervention was not performed in the remaining 17 patients (14.3\%). Pancreatoduodenectomy was performed in 54 patients, as well as total pancreatectomy in 8 patients and resection of the distal pancreas in 23 patients.

Combined resection of the PV or SMV was done in 26 patients, and tumor invasion of the vessel was histologically confirmed in 10 patients. Over $90 \%$ of the tumors were T3 or T4 according to both the JPS and UICC classifications. Sixty-six patients (55.5\%) had lymph node metastasis and 32 patients (26.9\%) had distant metastasis.

PALN dissection was performed in 71 patients (60.0\%), while it was not performed in the remaining 48 patients (40.0\%) because of liver metastasis, peritoneal dissemination, or malignant cells on abdominal lavage cytology. PALN metastasis was confirmed histologically in 6 patients (8.5\%), and lymph node metastasis was detected by intraoperative frozen section examination in 5 of them. In one patient, intraoperative examination failed to detect lymph node metastasis, but it was revealed by postoperative examination of formalin-fixed sections.

A comparison of clinical characteristics and tumor stage between the patients with and without PALN metastasis is presented in Table 3. It can be seen that the age, gender, tumor location, tumor size, and T factor of the patients with PALN metastasis were similar to those of the patients without 
metastasis. However, the $\mathrm{N}$ factor, $\mathrm{M}$ factor, and tumor stage (according to both the JPS and UICC classifications) differed between the two groups because the patients with PALN metastasis were classified as M1 and stage IV according to JPS.

\section{Diagnostic accuracy of preoperative imaging}

Assessment of the diagnostic accuracy of preoperative imaging studies was presented in Table 4. According to preoperative CT, 13 patients were suspected to have PALN metastasis, but metastasis was not found by histological examination in any of these patients. Similarly, 2 patients were suspected to have PALN metastasis on preoperative MRI and one patient by PET, but metastasis was not found in any of them on histological examination.

Finally, 6 patients were histologically diagnosed as having PALN metastasis, but these metastases were not found by any of the preoperative imaging studies (a sensitivity of $0 \%$ for each modality). In contrast to the low sensitivity, the specificity and accuracy were relatively high, being $79.4 \%$ and $72.5 \%$ for CT, $96.8 \%$ and $88.4 \%$ for MRI, and $98.4 \%$ and $90.0 \%$ for FDG-PET, respectively.

A comparison of lymph node size between patients with and without PALN metastasis is presented in Table 5. The longer diameter, shorter diameter, and ratio of the longer to shorter diameters of the PALN on imaging studies did not differ significantly between the two groups. The calculated volume of PALN with metastases was slightly larger than that of PALN without metastases, although the difference was not statistically significant. 


\section{Discussion}

Lymph node metastasis is one of the most important prognostic factors for gastrointestinal cancer, including pancreatic cancer. The PALNs are considered to be the final nodes that are invaded by periampullary and gastric cancers before the tumor enters the systemic lymphatic circulation. Accordingly, the majority of surgeons believe that metastases to these nodes represent systemic disease and they recommend that radical surgery (including extended lymphadenectomy) should not be done in patients with PALN metastasis ${ }^{4,12,13}$. Therefore, whether or not PALN metastasis exists is a major determinant of the surgical strategy.

Patients with PALN metastasis frequently also have distant metastases, such as liver involvement or peritoneal dissemination. If such distant metastases are not found, PALN metastasis is the final decider of whether or not a patient has resectable disease. The actual sensitivity of CT, MRI, and FDG-PET for diagnosis of PALN metastasis in patients with pancreatic cancer might be higher than that shown by the present study, because a large number of patients who were obviously not suitable for surgical resection due to distant metastasis were not included in this investigation. However, the fact that preoperative imaging studies could not detect histological PALN metastasis in any of our 6 patients should be emphasized, because it indicates that intraoperative frozen section examination is required to determine the treatment strategy for patients with pancreatic cancer even if preoperative imaging does not suggest PALN metastasis.

It remains controversial how to distinguish metastatic lymph nodes from normal lymph nodes on imaging studies. Lymph node size is most frequently used, but there is no precise criterion to differentiate benign from metastatic nodes. For example, the longer diameter, shorter diameter, or longer/shorter diameter ratio are frequently used parameters, but the reference values used differ between each report ${ }^{14,15}$. In patients with pancreatic cancer, imaging modalities show poor performance for detecting lymph node metastases and previous studies have shown that it is very 
hard to assess nodal involvement simply from the size or to determine the appropriate criterion ${ }^{7,16 \text {, }}$

17. Therefore, CT and MRI have a low priority with respect to identification of metastatic lymph nodes.

Previously, we reported that FDG-PET is not only useful for differentiating between pancreatic cancer and chronic pancreatitis but also for detecting small early pancreatic tumors ${ }^{18}$. It has been reported that the sensitivity of FDG-PET is $85 \%$ to $100 \%$ and the specificity is $53 \%$ to $100 \%$ for the diagnosis of pancreatic carcinoma ${ }^{18-22}$. However, the ability of FDG-PET to detect small metastases is quite limited and this imaging modality has not been found to significantly increase the accuracy of diagnosing metastasis ${ }^{8}$.

For identifying metastatic lymph nodes, it has been reported that FDG-PET is superior to morphologic imaging because it provides functional data, and thus has better sensitivity and specificity than $\mathrm{CT}^{23}$. However, technical limitations include relatively poor spatial resolution with diminished and/or incorrect anatomical localization, as well as missing small spots of increased tracer uptake. Thus, the actual sensitivity of FDG-PET for lymph node metastasis of pancreatic cancer has been reported to be between $46 \%$ and $71 \%$, with a specificity ranging between $63 \%$ and $100 \%{ }^{24-28}$. Although it is true that patients with obviously unresectable disease were not included in our study, so the true sensitivity and specificity of FDG-PET would be expected to be higher than shown by our results, FDG-PET data would not change the clinical management of the vast majority of patients previously evaluated by CT.

To obtain more accurate and simpler methods for the diagnosis of PALN metastasis, further development of diagnostic modalities will be necessary. Diffusion-weighted MRI (DWI), MRI using lymphotropic contrast agents, and combined PET-CT imaging seem to be the most promising modalities currently available for accurate lymph node evaluation ${ }^{24,29}$. Although further 
technical advances are necessary to improve spatial resolution and to reduce susceptibility and motion artifacts, it is expected that it may become possible to characterize even small lymph nodes as benign or malignant in the future ${ }^{30}$.

For example, dextran-coated ultra-small superparamagnetic iron oxide particles (USPIOs) have previously been evaluated for specifically enhancing lymphatic tissues and for discriminating between malignant and benign lymph nodes ${ }^{24}$. Recently, lymphotrophic nanoparticle-enhanced MRI (LNMRI) has emerged has a promising imaging tool for lymph nodes, and it has been reported that LNMRI has a significantly higher sensitivity and negative predictive value than CT ${ }^{31,32}$.

Moreover, other new MRI contrast agents, such as T1-enhancing contrast agents and targeted MR contrast agents have been also evaluated for lymph node imaging ${ }^{24}$. Combined ET-CT employs the functional physiological information obtained by FDG-PET and the detailed anatomical information provided by CT to localize diseased lymph nodes more specifically than can be done with a single imaging modality ${ }^{23,24}$; however, differentiating inflammation from malignancy and detecting microscopic metastases must still be considered as limitations of PET-CT 33.

In conclusion, preoperative detection of PALN metastasis is difficult in patients who are considered to be candidates for resection of pancreatic cancer and intraoperative histology is absolutely necessary. It seems that discrimination between benign and malignant lymph nodes by current imaging modalities is almost impossible, and further development of imaging methods that include functional and qualitative assessment may be necessary. Until then, intraoperative frozen section examination of PALN is recommended before other surgical procedures are performed in patients with pancreatic cancer. 


\section{References}

1. Matsuno S, Egawa S, Fukuyama S, Motoi F, Sunamura M, Isaji S, et al. Pancreatic Cancer Registry in Japan: 20 years of experience. Pancreas 2004; 28:219-30.

2. Herrmann R, Jelic S. Pancreatic cancer: ESMO clinical recommendations for diagnosis, treatment and follow-up. Ann Oncol 2008; 19 Suppl 2:ii25-6.

3. Riall TS, Cameron JL, Lillemoe KD, Campbell KA, Sauter PK, Coleman J, et al. Pancreaticoduodenectomy with or without distal gastrectomy and extended retroperitoneal lymphadenectomy for periampullary adenocarcinoma--part 3: update on 5-year survival. $\mathbf{J}$ Gastrointest Surg 2005; 9:1191-204; discussion 204-6.

4. Doi R, Kami K, Ito D, Fujimoto K, Kawaguchi Y, Wada M, et al. Prognostic implication of para-aortic lymph node metastasis in resectable pancreatic cancer. World J Surg 2007; 31:147-54.

5. Kayahara M, Nagakawa T, Ohta T, Kitagawa H, Ueno K, Tajima H, et al. Analysis of paraaortic lymph node involvement in pancreatic carcinoma: a significant indication for surgery? Cancer 1999; 85:583-90.

6. Schwarz M, Pauls S, Sokiranski R, Brambs HJ, Glasbrenner B, Adler G, et al. Is a preoperative multidiagnostic approach to predict surgical resectability of periampullary tumors still effective? Am J Surg 2001; 182:243-9.

7. Soriano A, Castells A, Ayuso C, Ayuso JR, de Caralt MT, Gines MA, et al. Preoperative staging and tumor resectability assessment of pancreatic cancer: prospective study comparing endoscopic ultrasonography, helical computed tomography, magnetic resonance imaging, and angiography. Am J Gastroenterol 2004; 99:492-501.

8. Parsons CM, Sutcliffe JL, Bold RJ. Preoperative evaluation of pancreatic adenocarcinoma. J Hepatobiliary Pancreat Surg 2008; 15:429-35. 
9. Japan Pancreas Society. Classification of pancreatic carcinoma (2nd English edition). Tokyo: Kanehara \& Co., 2003.

10. Sobin LH, Wittekind C. TNM classification of malignant tumours 6th edition. New York: Wiley-Liss, 2002.

11. Committee on Classification of Regional Lymph Nodes of Japan Society of Clinical Oncology. Classification of regional lymph nodes in Japan. Int J Clin Oncol 2003; 8:248-75.

12. Yoshida T, Matsumoto T, Sasaki A, Shibata K, Aramaki M, Kitano S. Outcome of paraaortic node-positive pancreatic head and bile duct adenocarcinoma. Am J Surg 2004; 187:736-40.

13. Shimada K, Sakamoto Y, Sano T, Kosuge T. The role of paraaortic lymph node involvement on early recurrence and survival after macroscopic curative resection with extended lymphadenectomy for pancreatic carcinoma. J Am Coll Surg 2006; 203:345-52.

14. de Bondt RB, Nelemans PJ, Hofman PA, Casselman JW, Kremer B, van Engelshoven JM, et al. Detection of lymph node metastases in head and neck cancer: a meta-analysis comparing US, USgFNAC, CT and MR imaging. Eur J Radiol 2007; 64:266-72.

15. Hovels AM, Heesakkers RA, Adang EM, Jager GJ, Strum S, Hoogeveen YL, et al. The diagnostic accuracy of CT and MRI in the staging of pelvic lymph nodes in patients with prostate cancer: a meta-analysis. Clin Radiol 2008; 63:387-95.

16. Karmazanovsky G, Fedorov V, Kubyshkin V, Kotchatkov A. Pancreatic head cancer: accuracy of CT in determination of resectability. Abdom Imaging 2005; 30:488-500.

17. Roche CJ, Hughes ML, Garvey CJ, Campbell F, White DA, Jones L, et al. CT and pathologic assessment of prospective nodal staging in patients with ductal adenocarcinoma of the head of the pancreas. AJR Am J Roentgenol 2003; 180:475-80.

18. Seo S, Doi R, Machimoto T, Kami K, Masui T, Hatano E, et al. Contribution of 
${ }^{18}$ F-fluorodeoxyglucose positron emission tomography to the diagnosis of early pancreatic carcinoma. J Hepatobiliary Pancreat Surg 2008; 15:634-9.

19. Rose DM, Delbeke D, Beauchamp RD, Chapman WC, Sandler MP, Sharp KW, et al. ${ }^{18}$ Fluorodeoxyglucose-positron emission tomography in the management of patients with suspected pancreatic cancer. Ann Surg 1999; 229:729-37; discussion 37-8.

20. Berberat P, Friess H, Kashiwagi M, Beger HG, Buchler MW. Diagnosis and staging of pancreatic cancer by positron emission tomography. World J Surg 1999; 23:882-7.

21. Sperti C, Pasquali C, Chierichetti F, Liessi G, Ferlin G, Pedrazzoli S. Value of 18-fluorodeoxyglucose positron emission tomography in the management of patients with cystic tumors of the pancreas. Ann Surg 2001; 234:675-80.

22. Imdahl A, Nitzsche E, Krautmann F, Hogerle S, Boos S, Einert A, et al. Evaluation of positron emission tomography with 2-[ $\left.{ }^{18} \mathrm{~F}\right]$ fluoro-2-deoxy-D-glucose for the differentiation of chronic pancreatitis and pancreatic cancer. Br J Surg 1999; 86:194-9.

23. Golder WA. Lymph node diagnosis in oncologic imaging: a dilemma still waiting to be solved. Onkologie 2004; 27:194-9.

24. Wunderbaldinger P. Problems and prospects of modern lymph node imaging. Eur J Radiol 2006; 58:325-37.

25. Bares R, Dohmen BM, Cremerius U, Fass J, Teusch M, Bull U. [Results of positron emission tomography with fluorine-18 labeled fluorodeoxyglucose in differential diagnosis and staging of pancreatic carcinoma]. Radiologe 1996; 36:435-40.

26. Bares R, Klever P, Hauptmann S, Hellwig D, Fass J, Cremerius U, et al. F-18 fluorodeoxyglucose PET in vivo evaluation of pancreatic glucose metabolism for detection of pancreatic cancer. Radiology 1994; 192:79-86.

27. Diederichs CG, Staib L, Vogel J, Glasbrenner B, Glatting G, Brambs HJ, et al. Values and 
limitations of ${ }^{18}$ F-fluorodeoxyglucose-positron-emission tomography with preoperative evaluation of patients with pancreatic masses. Pancreas 2000; 20:109-16.

28. Pakzad F, Groves AM, Ell PJ. The role of positron emission tomography in the management of pancreatic cancer. Semin Nucl Med 2006; 36:248-56.

29. Kim JK, Kim KA, Park BW, Kim N, Cho KS. Feasibility of diffusion-weighted imaging in the differentiation of metastatic from nonmetastatic lymph nodes: early experience. J Magn Reson Imaging 2008; 28:714-9.

30. Holzapfel K, Duetsch S, Fauser C, Eiber M, Rummeny EJ, Gaa J. Value of diffusion-weighted MR imaging in the differentiation between benign and malignant cervical lymph nodes. Eur J Radiol 2008; (online first).

31. Saokar A, Braschi M, Harisinghani M. Lymphotrophic nanoparticle enhanced MR imaging (LNMRI) for lymph node imaging. Abdom Imaging 2006; 31:660-7.

32. Heesakkers RA, Hovels AM, Jager GJ, van den Bosch HC, Witjes JA, Raat HP, et al. MRI with a lymph-node-specific contrast agent as an alternative to CT scan and lymph-node dissection in patients with prostate cancer: a prospective multicohort study. Lancet Oncol 2008; 9:850-6.

33. Veit P, Ruehm S, Kuehl H, Stergar H, Mueller S, Bockisch A, et al. Lymph node staging with dual-modality PET/CT: enhancing the diagnostic accuracy in oncology. Eur J Radiol 2006; 58:383-9. 

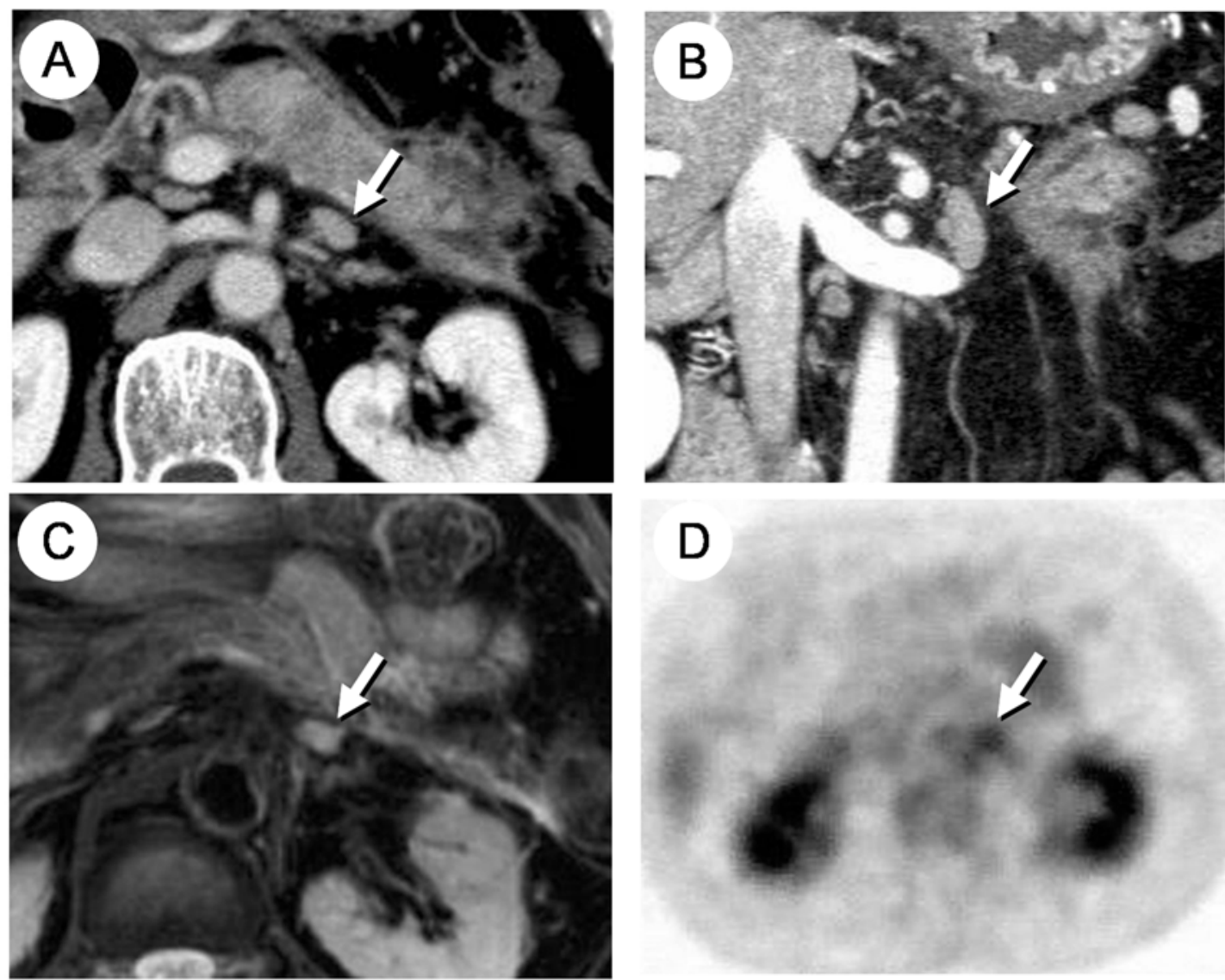

D

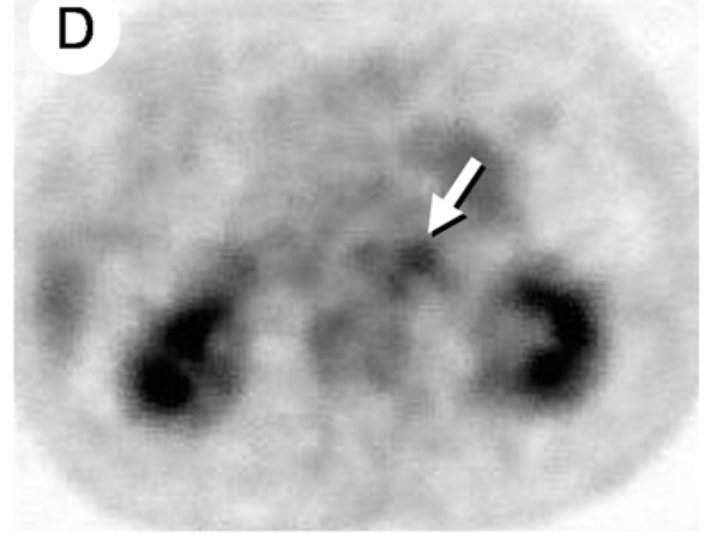

Figure 1. Preoperative imaging studies.

Computed tomography (A and B) and magnetic resonance imaging (C) show an ovoid 13-mm para-aortic lymph node (arrows). $\quad{ }^{18}$ F-fluorodeoxyglucose uptake by a para-aortic lymph node (arrow) on a positron emission tomography image (D). 
$-19-$

\section{Table 1.}

Clinical, surgical, and histopathological findings of 119 patients

\begin{tabular}{llc}
\hline Age (years, mean \pm SD [range]) & & $65.0 \pm 9.3[32-85]$ \\
Gender & Male & $58(48.7 \%)$ \\
& Female & $61(51.3 \%)$ \\
Location & Head & $79(66.4 \%)$ \\
& Body & $23(19.3 \%)$ \\
& Tail & $5(4.2 \%)$ \\
& Head + body & $1(0.8 \%)$ \\
Bumor size (mm, mean \pm SD [range]) & $10(8,4 \%)$ \\
Surgery & PD, PPPD & $1(0.8 \%)$ \\
& DP & $31.3 \pm 15.6[8-120]$ \\
TP & $54(45.4 \%)$ \\
PALN dissection & Palliation & $23(19.3 \%)$ \\
& Probe laparotomy & $8(6.7 \%)$ \\
& & $17(14.3 \%)$ \\
& & $17(14.3 \%)$ \\
\hline
\end{tabular}

PD, pancreaticoduodenectomy; PPPD, pylorus-preserving pancreaticoduodenectomy; DP, distal pancreatectomy; TP, total pancreatectomy; PALN, para-aortic lymph node; PV, portal vein; SMV, superior mesenteric vein. 
$-20-$

\section{Table 2.}

Staging of 119 patients by JPS and UICC classification

\begin{tabular}{llc}
\hline JPA Stage & I & $1(0.8 \%)$ \\
II & $2(1.7 \%)$ \\
III & $21(17.6 \%)$ \\
IV a & $59(50.0 \%)$ \\
IV b & $36(30.3 \%)$ \\
UICC Stage & Ia & $1(0.8 \%)$ \\
Ib & $2(1.7 \%)$ \\
IIa & $36(30.3 \%)$ \\
IIb & $44(37.0 \%)$ \\
III & $4(3.4 \%)$ \\
IV & $32(26.9 \%)$
\end{tabular}

JPS, Japan Pancreas Society; UICC, Union International Contra la Cancrum 
$-21-$

\section{Table 3.}

Comparison of clinical characteristics and tumor staging between patients with and without para-aortic lymph node metastasis.

\begin{tabular}{|c|c|c|c|c|}
\hline & & $\begin{array}{l}\text { PALN metastasis } \\
(+) \quad(\mathrm{n}=6)\end{array}$ & $\begin{array}{l}\text { PALN metastasis } \\
(-) \quad(n=65)\end{array}$ & $P$ value \\
\hline Age & & $65.5 \pm 6.2$ & $63.5 \pm 10.1$ & 0.63 \\
\hline \multirow[t]{2}{*}{ Gender } & Male & $3(50 \%)$ & $30(46.2 \%)$ & 0.83 \\
\hline & Female & $3(50 \%)$ & 35 (53.8\%) & \\
\hline \multirow[t]{5}{*}{ Location } & Head & 5 (83.3\%) & 47 (72.3\%) & 0.79 \\
\hline & Body & $1(16.7 \%)$ & $9(13.8 \%)$ & \\
\hline & Tail & 0 & $1(1.5 \%)$ & \\
\hline & Head / body & 0 & $1(1.5 \%)$ & \\
\hline & Body / tail & 0 & 7 (10.8\%) & \\
\hline Tumor size (mm) & & $24.4 \pm 3.9$ & $30.2 \pm 14.0$ & 0.31 \\
\hline \multirow[t]{5}{*}{ JPS Stage } & I & 0 & $1(1.5 \%)$ & $<0.001$ \\
\hline & II & 0 & $2(3.1 \%)$ & \\
\hline & III & 0 & $15(23.1 \%)$ & \\
\hline & IV a & 0 & $42(64.6 \%)$ & \\
\hline & IV b & $6(100 \%)$ & $11(16.9 \%)$ & \\
\hline \multirow[t]{6}{*}{ UICC Stage } & Ia & 0 & $1(1.5 \%)$ & $<0.001$ \\
\hline & $\mathrm{Ib}$ & 0 & $2(3.1 \%)$ & \\
\hline & IIa & 0 & $23(35.4 \%)$ & \\
\hline & IIb & 0 & 35 (53.8\%) & \\
\hline & III & 0 & $1(1.5 \%)$ & \\
\hline & IV & $6(100 \%)$ & $3(4.6 \%)$ & \\
\hline
\end{tabular}

Age and tumor size are expressed as the mean \pm SD.

PALN, para-aortic lymph node; JPS, Japan Pancreas Society; UICC, Union International Contra la Cancrum 
Table 4.

Diagnostic accuracy of preoperative imaging studies.

\begin{tabular}{lccccc}
\hline & $\begin{array}{c}\text { No. of } \\
\text { patients }\end{array}$ & $\begin{array}{c}\text { Patients with suspected } \\
\text { PALN metastasis }\end{array}$ & Sensitivity (\%) & $\begin{array}{c}\text { Specificity } \\
(\%)\end{array}$ & $\begin{array}{c}\text { Accuracy } \\
(\%)\end{array}$ \\
\hline CT & 69 & 13 & 0 & $79.4 \%$ & $72.5 \%$ \\
MRI & 69 & 2 & 0 & $96.8 \%$ & $88.4 \%$ \\
FDG-PET & 69 & 1 & 0 & $98.4 \%$ & $90.0 \%$ \\
\hline
\end{tabular}

CT, computed tomography; MRI, magnetic resonance imaging; PET, positoron emission tomography; PALN, para-aortic lymph node. 
Table 5.

Comparison of lymph node size parameters.

\begin{tabular}{|c|c|c|c|}
\hline & $\begin{array}{l}\text { PALN metastasis } \\
(+) \quad(\mathrm{n}=6)\end{array}$ & $\begin{array}{l}\text { PALN metastasis } \\
(-) \quad(\mathrm{n}=65)\end{array}$ & $\begin{array}{c}P \\
\text { value }\end{array}$ \\
\hline Longer diameter (mm) & $8.1 \pm 1.0$ & $7.4 \pm 2.2$ & 0.42 \\
\hline Shorter diameter (mm) & $5.0 \pm 1.1$ & $4.6 \pm 1.5$ & 0.56 \\
\hline Long/Short ratio & $1.7 \pm 0.4$ & $1.7 \pm 0.4$ & 0.80 \\
\hline Calculated volume $\left(\mathrm{mm}^{3}\right)$ & $122 \pm 50$ & $99 \pm 85$ & 0.54 \\
\hline
\end{tabular}

PALN, para-aortic lymph node

Data are expressed as the mean $\pm \mathrm{SD}$. 